\title{
The ecocultural context and child behavior problems: A qualitative analysis in rural Nepal
}

\author{
Matthew D. Burkey ${ }^{a,}{ }^{*}$, Lajina Ghimire ${ }^{b}$, Ramesh Prasad Adhikari ${ }^{b}$, Lawrence S. Wissow ${ }^{c}$, \\ Mark J.D. Jordans ${ }^{\mathrm{d}}$, Brandon A. Kohrt ${ }^{\mathrm{e}}$ \\ a Johns Hopkins School of Public Health, Department of Health, Behavior and Society, 624 N. Broadway, Hampton House 703, Baltimore, MD 21205, USA \\ ${ }^{\mathrm{b}}$ Transcultural Psychosocial Organization-Nepal, Baluwatar, Kathmandu, Nepal \\ c Johns Hopkins Bloomberg School of Public Health, 624 N. Broadway, Baltimore, MD 21205, USA \\ d War Child Holland, Helmholtzstraat 61-G, 1098 LE Amsterdam, The Netherlands \\ e Duke Global Health Institute, 310 Trent Dr, Durham, NC 27710, USA
}

\section{A R T I C L E I N F O}

\section{Article history:}

Received 30 November 2015

Received in revised form 12 April 2016

Accepted 14 April 2016

Available online 3 May 2016

\section{Keywords:}

Nepal

Child development

Culture

Attention deficit and disruptive behavior

disorders

Qualitative research

\begin{abstract}
A B S T R A C T
Commonly used paradigms for studying child psychopathology emphasize individual-level factors and often neglect the role of context in shaping risk and protective factors among children, families, and communities. To address this gap, we evaluated influences of ecocultural contextual factors on definitions, development of, and responses to child behavior problems and examined how contextual knowledge can inform culturally responsive interventions. We drew on Super and Harkness' "developmental niche" framework to evaluate the influences of physical and social settings, childcare customs and practices, and parental ethnotheories on the definitions, development of, and responses to child behavior problems in a community in rural Nepal. Data were collected between February and October 2014 through in-depth interviews with a purposive sampling strategy targeting parents $(\mathrm{N}=10)$, teachers $(\mathrm{N}=6)$, and community leaders $(\mathrm{N}=8)$ familiar with child-rearing. Results were supplemented by focus group discussions with children $(\mathrm{N}=9)$ and teachers $(\mathrm{N}=8)$, pile-sort interviews with mothers $(\mathrm{N}=8)$ of school-aged children, and direct observations in homes, schools, and community spaces. Behavior problems were largely defined in light of parents' socialization goals and role expectations for children. Certain physical settings and times were seen to carry greater risk for problematic behavior when children were unsupervised. Parents and other adults attempted to mitigate behavior problems by supervising them and their social interactions, providing for their physical needs, educating them, and through a shared verbal reminding strategy (samjhaune). The findings of our study illustrate the transactional nature of behavior problem development that involves context-specific goals, roles, and concerns that are likely to affect adults' interpretations and responses to children's behavior. Ultimately, employing a developmental niche framework will elucidate setting-specific risk and protective factors for culturally compelling intervention strategies.
\end{abstract}

() 2016 Elsevier Ltd. All rights reserved.

\footnotetext{
* Corresponding author. Johns Hopkins School of Public Health, 624 N. Broadway, Hampton House 703, Baltimore, MD 21205, USA.

E-mail addresses: mburkey1@jhmi.edu (M.D. Burkey), lajina538@gmail.com (L. Ghimire), radhikari@tponepal.org.np (R.P. Adhikari), lwissow@jhmi.edu (L.S. Wissow), mark.jordans@warchild.nl (M.J.D. Jordans), brandon.kohrt@duke. edu (B.A. Kohrt).
}

\section{Introduction}

\subsection{Background}

Definitions of and responses to behavior problems are highly dependent on the context in which they occur. All child behavior takes place within a particular physical and social setting, and elicits responses from caregivers that are shaped by their customs and beliefs (Super and Harkness, 1986). More broadly, child development occurs as a transactional process situated within social, ecological, and cultural contexts (referred to here as 
“ecocultural contexts”) (Bronfenbrenner, 1979, 2005; Harkness and Super, 1996; Sameroff, 2009; Super and Harkness, 1986; Worthman, 2010a, 2010b; Zilanawala et al., 2015). Yet, contemporary scholarship in the field of psychiatry often proceeds with the implicit assumption that psychopathologies in children can be identified, understood, and effectively treated with minimal consideration of the child's contexts. As a result, psychiatric definitions and treatments often lack coherence (i.e. understandability) or relevance to local concerns and are met with limited engagement when applied in novel ecocultural contexts (Kleinman, 1977; Lau, 2006).

Whereas widely used clinical and research definitions focus attention on differences in amount of specified behavior problems between contexts, the types of symptoms or behaviors that are problematic in one ecocultural setting may have substantially different meaning or significance in other settings (Kirmayer and Swartz, 2013). Culturally rooted symbolic meanings may affect the course and outcome of emotional and behavioral problems by shaping interpersonal responses to affected individuals, including family coping processes and patient-provider interactions (Kirmayer and Swartz, 2013). Weisz et al. (1997) ascribe similar importance to the context-dependent meaning of symptoms: "child psychopathology is inevitably the study of two phenomena-the behavior of children and the lens through which adults view child behavior" (p. 569). For example, Lambert et al. (1992) found that when parents in Jamaica and the U.S. evaluated similar patterns of child behavior (in vignettes) they had different "thresholds" for concern, were concerned about different aspects of behavior, and had different expectations for future course.

Cultural anthropologists have described cultural contexts of parenting and child development (LeVine and New, 2008; Worthman, 2010a, 2010b), though little anthropological scholarship has focused specifically on the topic of child behavior problems. In contrast, clinically focused studies of child behavior problems in non-Western settings have rarely considered the symbolic meaning or local relevance of symptoms. Instead, crossnational epidemiologic studies have focused primarily on quantifying the frequency or severity of pre-specified sets of behavior problems using standardized assessment tools, and have found similar rates of diagnoses and problem patterns (c.f. Canino et al., 2010; Crijnen et al., 1997; Kessler et al., 2007). These studies primarily draw on causal frameworks related to endogenous child factors or operant conditioning that are supposedly universally related to psychopathology.

One of the leading models for understanding the development of child behavior problems is Patterson's (2002) "coercive family process" model rooted in social learning theory. Patterson's model focuses on child-parent behavioral interactions in which children's non-compliance is reinforced by escape and/or parents' escalating punishments are reinforced by compliance. While Patterson's model has formed a useful foundation for predicting trajectories of noncompliance and has informed parenting interventions, it does not distinguish which forms of non-compliance are more or less concerning to parents, address how parent-child interactions are shaped by the ecocultural context, or elucidate extra-familial factors shaping child behavior problems.

Relatively little attention has focused on the ecocultural context of parenting and child development, in which behaviors may be variously defined by local stakeholders as "normal" or problematic. This is an important area for research because most widely used definitions (American Psychiatric Association, 2013; World Health Organization, 2010), clinical assessment tools (Achenbach and Edelbrock, 1983; Goodman, 1997), and clinical interventions (Furlong et al., 2012; Woolfenden et al., 2001) have been developed in Western settings but are increasingly applied in vastly different settings (e.g., low-income, non-Western countries). Systematic reviews indicate that $94-96 \%$ of published studies in psychology and psychiatry have taken place in high-income, Western countries (Arnett, 2008; V. Patel and Sumathipala, 2001).

\subsection{The developmental niche as a framework for studying culture and behavior problems}

In contrast to psychopathological models that view endogenous child-level biological factors as deterministic and "universal", ecologically-focused developmental psychologists and anthropologists suggest the "child-in-context" as a more appropriate object of study (Super and Harkness, 1986). Ecological theorists posit that biological predispositions are continuously shaped throughout development by macro-social factors acting via their influences on the proximal conditions of child development (Whiting, 1977; Worthman, 2010b). Similarly, ecological models of child development situate parent's expectations and childcare practices within a rich theoretical framework that relates higher-level cultural factors with child-rearing practices (Super and Harkness, 1986; Worthman, 2010a, 2010b).

In this study, we drew upon Super and Harkness' (Super and Harkness, 1986, 2002) concept of the "developmental niche" as a useful framework to study how higher-order influences structure the environment for child development. The developmental niche is described as the composite of three subsystems: 1) the physical and social settings in which the child lives, 2) childcare customs and practices, and 3) parents' psychology (or beliefs). Together, these three subsystems interact to influence child development over time. In Super and Harkness' model, "physical and social settings" refer to characteristics of the physical places where children spend time, the people they spend time with, and the social roles (e.g., work vs. play) children fill throughout the day. "Childcare customs and practices" refer to sequences of behavior that are commonly used and accepted when interacting with children in given situations or stages of development. "Parents' psychology" refers to the beliefs that commonly accompany childcare customs and include "beliefs concerning the nature and needs of children, parental and community goals for childrearing, and caretaker beliefs about effective rearing techniques" (i.e. "ethnotheories") (Super and Harkness, 1986, p. 556). Together, these three "subsystems" mediate the child's experience within her/his culture throughout development and result in the child learning the rules of the culture.

\subsection{Aims of this study}

Our aim in this study was to describe the "developmental niche" in a community in rural Nepal and identify the influences of physical and social settings, caregiver practices, and parental ethnotheories on definitions, development of, and responses to child behavior problems. We provide a description of all three subsystems of the developmental niche, focusing primarily on understanding parents' and teachers' goals for children, expectations of child behavior, and concepts of behavior problems, including mitigation strategies. We selected an initial focus on parents and teachers because they disproportionately shape the everyday environments of children at home and in classrooms throughout the early school years, and-as gatekeepers to treatment-understanding their perceptions and ideas is critical to developing acceptable and relevant interventions.

\subsection{Study setting: Nepal}

As a low-income country in South Asia emerging from a decade- 
long civil war (United Nations Development Programme [UNDP], 2009), Nepal differs along socioeconomic, political, and key cultural dimensions (e.g., language, religion) from the contexts in which clinical constructs of child mental disorders developed and have primarily been studied (i.e., the U.S. and Western Europe). Nepal is a majority Hindu nation (81\%) with a substantial Buddhist minority (8\%) and a great deal of ethnic, linguistic, and socioeconomic diversity, including 125 caste or ethnic groups reported in the most recent census (Central Bureau of Statistics (2011)). By conducting this study in Nepal, we offer a novel perspective on the potential variability in stakeholders' concepts of behavior problems. In addition, Nepal is a suitable place to study child behavior problems and context, as it has been the site of prior research in fields related to culture and child development, including: parents' goals for socialization of affective displays (Cole and Tamang, 1998; Cole et al., 2006), socioeconomic determinants of child neurodevelopmental outcomes (S. A. Patel et al., 2013), and ethnopsychological models of children's emotional and behavioral transition to adulthood in the context of domestic and political violence (Kohrt \& Maharjan, 2009). Therefore, our study builds on existing scholarship to develop a more detailed view of child behavioral development in context, while adding a focus on behavior problems and mitigation strategies.

\section{Methods}

\subsection{Research setting and study site}

The current study took place in the Chitwan District of the south-central lowlands (Terai) region of Nepal. Meghauli, one of 40 Village Development Committees (VDCs, the smallest administrative unit in Nepal) in Chitwan District, was selected as the site for this study because it is the setting for a recent primary care/mental health integrated care delivery project (Lund et al., 2012). This project was conducted as part of the formative research to extend the existing project to include contextually relevant services for children. (See "Results" for additional details about the study setting.)

\subsection{Sampling and participants}

The sampling for this study was purposive. Participants were selected based on the goals of identifying participants who: (1) were familiar with childhood and childrearing in the community, and (2) represented a wide range of roles and perspectives with respect to child behavior. Specifically, we sought to include parents, teachers, community leaders, and children of both genders and with varying educational, caste, and socioeconomic backgrounds. Local liaisons (female community health volunteers working in the formal public healthcare delivery system) from throughout Meghauli assisted the research team in identifying and recruiting parents, community leaders, and children from their communities who met the sampling goals. School principals also assisted in identifying a diverse sample of knowledgeable teachers for interviews and focus groups. Interview participants were asked to identify other community members who were knowledgeable about childhood and childrearing, and these individuals were approached for participation. Children (ages 7-15) were included as participants in focus group discussions. Participants were informed that the study goals were to understand the situations and challenges of children and families in the local community in order to develop a useful, supportive intervention.

\subsection{Data collection}

Data were collected between February and October 2014 using a combination of qualitative research methods. The timing of data collection over a 9-month period and conducting interviews on a combination of weekdays and weekends during daytime and evening hours allowed greater access to informants with seasonal work or longer work hours.

The primary data collection method was in-depth interviews using semi-structured interview guides. This data was supplemented by focus group discussions, pile-sorting interviews, field observations, and a focused archival review. Interviews and focus groups were conducted by a Nepali researcher (second author) who received initial training and ongoing supervision (via weekly calls or in-person meetings) from a Nepali mental health research supervisor (third author) and a child psychiatrist/public health researcher from the United States (first author). All interviews and focus groups were conducted in colloquial Nepali. Interviews took place in private locations; most interviews were conducted in participants' homes, or for teachers, in the schools where they worked.

\subsubsection{In-depth interviews}

We conducted 24 in-depth interviews with parents $(\mathrm{N}=10)$, teachers $(N=6)$, and other community leaders $(N=8)$. The sample of interviewees included 11 women and 13 men, including 10 highcaste Hindus, 4 low-caste Hindus, and 8 members of other ethnic groups (Tamang, Tharu, Kumal, and Magar). The informants came from a variety of occupations (e.g., principal, government workers, teachers, farmers, homemakers, foreign worker, and driver) and had a range of educational backgrounds (no formal education to master's degree). The interview guide focused on eliciting information about physical and social settings of child development (e.g. children's daily routines and social roles and the people they are surrounded by); childcare customs and practices (e.g., responsibility for childcare roles, common caregiver responses to child misbehavior); and ethnotheories (i.e. the "nature and needs" of children, goals for childrearing, and concepts about effective rearing techniques) (Super and Harkness, 1986). The interview guide also specifically assessed key beliefs related to child behavior problems, including: causes, associated symptoms, expected course, and effective mitigation strategies.

The interviews typically lasted 30-90 min. We returned to several of the more informative participants on multiple occasions to ask focused follow-up questions as needed over the course of 9 months and took focused hand-written notes during these meetings. The most informative participants were contacted approximately monthly during the study period.

\subsubsection{Vignette-based focus group discussions (FGDs)}

We conducted four FGDs with a total of 17 participants. FGDs were held separately with teachers ( $\mathrm{N}=8 ; 50 \%$ female) and children $(\mathrm{N}=9 ; 44 \%$ female), and the groups were divided by gender of the participants. Discussions were audio recorded and began with a brief vignette describing an 11-year-old child exhibiting a variety of behavior-related problems (e.g., arguing, fighting, non-compliance with teachers' directives). Participants were asked to comment on which parts of the child's behavior were most concerning to them, potential causes, plausible course and long-term outcomes, and helpful treatments.

\subsubsection{Pile-sorting interviews}

Pile-sorting interviews were conducted with mothers $(\mathrm{N}=8)$ of study-age children using cards (with word and picture cues) with ten behavior problems taken from in-depth interviews and free- 
listing exercises (from a previous study conducted in the same community (Adhikari et al., 2015). The purpose of pile-sorting interviews was to understand cognitive domains (Borgatti, 1999) related to behavior problems and factors affecting perceived severity of behavior problems. Participants were first asked to sort the cards into $2-3$ piles in any way that made sense to them, then in order of severity. Participants were asked to explain the reasons they sorted the cards as they did. We recorded the orders and groupings of their sorts, as well as the explanations provided.

\subsubsection{Field notes and observations}

The first and second authors also observed and made handwritten field notes of children's activities, interactions, and behaviors during and immediately after in-home interviews, in public spaces (including community gathering places and along roadsides), and during visits to three area primary and secondary schools ( 2 public, 1 private). The main purpose of direct observations was to gain additional information about observable aspects of the developmental niche in the study community, focusing on where and with whom children spend time (i.e. physical and social settings) and to triangulate interview data. Observations were conducted on four separate visits over a period lasting 9 months. Field notes were typed in English and coded (as below).

\subsection{Research team and reflexivity}

The study was primarily designed and analyzed by the first author, a non-Nepali man from the United States trained as a child and adolescent psychiatrist and public health researcher. The interviews were conducted by the second author, a Nepali woman educated as a nurse and undergoing graduate training in psychology, who also assisted with interpreting and analyzing the data through discussions of interviews and observations in the field. Interviewees could tell from the interviewer's name that she was from one of the traditional "high" castes, and could usually tell from her appearance (e.g., clothing style) and through direct questioning that she was from an urban area and younger than most of the adults she interviewed. As the most visible face of the study, her apparent identity features were likely to have influenced participants' responses through their judgments of what a young, urban, educated, high-caste woman would find acceptable (i.e. social desirability).

Through discussions during the initial stages of data analysis between the first and second authors, it became apparent that different aspects of the interviews seemed salient to each analyst. The first author was most interested in settings, concepts, and child-rearing practices that contrasted to those familiar from his upbringing, his own parenting experience, and his clinical practice in an urban setting in the United States. The second author pointed out differences between what she heard and observed in the study community and her experiences growing up and practicing as a public health nurse in other areas in Nepal. These differences in experience and perspective resulted in discussions during data analysis that yielded a more thorough and comprehensive view of the data (e.g., a second look at a section originally de-emphasized by one analyst), offered points of comparison with other Nepali communities and with non-Nepali clinical and non-clinical settings, and offered a check to over-interpretation during analysis.

\subsection{Ethics}

This study was approved by the Institutional Review Board at the Johns Hopkins Bloomberg School of Public Health in the U.S. and by the Nepal Health Research Council. All adult participants provided oral consent and children provided assent. Compensation in the form of small household items (approximate value: US\$2-3) was provided to participants as determined by consultation with local researchers and the ethical review board in Nepal.

\subsection{Data preparation}

Audio recordings from interviews and focus group discussions were translated and transcribed into English. Key terms or phrases with ambiguous translations were retained in Nepali in the written transcripts. Transcriptions were spot-checked for quality by an anthropologist fluent in Nepali and English. All transcripts and typed field notes from direct observations were entered into the NVivo software platform (QSR International, 2012), which supported qualitative data analysis.

\subsection{Data analysis}

An initial codebook (including code labels, definitions, inclusion/exclusion criteria, and examples) was developed using predetermined codes based on the study objectives and research questions. Through reading initial transcripts, the authors defined additional emergent codes to address themes connecting and modifying the original research questions. The updated codebook was reviewed and edited through discussion among four of the authors. Using line-by-line coding in NVivo, the first author then applied codes to the transcripts and field notes.

Codes were then grouped into themes related to the study research questions. Through tabulating results, writing memos during coding, and discussions among team members, the authors synthesized the themes into categories related to subsystems of the developmental niche, their interconnections, and cross-cutting themes.

\subsection{Discussion of rigor and robustness}

We sought to enhance the credibility of our analysis through return visits, checking emerging concepts with key informants (e.g., a school principal, parents, female community health volunteers-all of whom were also interviewed), and peer debriefing with young mental health researchers raised near the study community and an anthropologist/psychiatrist with extensive experience in Nepal. We also made multiple visits to the community before, during, and after the formal research period in efforts to prolong our engagement with the study community and seek their input at multiple points in our research.

We incorporated methodologic triangulation into our study design and analysis in order to enhance the completeness of our report and "enlarg(e) the landscape of (our) inquiry" (Tobin and Begley, 2004, p. 393). Our goal in utilizing multiple methods of investigation was primarily to evaluate for multiple understandings of our study topic (i.e. completeness) by including apparently contradictory viewpoints, exceptions, and nuances. A secondary goal was to corroborate data collected from one source by comparing it with data from other sources (i.e. verification).

\section{Results}

\subsection{Overview of codes and categories}

The codes were organized into six categories representing the three subsystems of the developmental niche, defining behavior problems, development of behavior problems, and mitigating behavior problems. Analysis of how settings, practices, and beliefs related to defining, developing, and responding to behavior problems provided the framework for the results presented below. 
Findings are summarized in Fig. 1.

\subsection{Overview of the developmental niche in Meghauli}

\subsubsection{Physical and social settings}

Meghauli VDC is primarily rural, set at a 1- to 2-h bus ride from Bharatpur (population 199,867) (Central Bureau of Statistics (2011)). Meghauli is shaped like a triangle, surrounded on two sides by the Narayani River and the East Rapti River. Most of the land in Meghauli is dedicated to fields growing rice and other grains. A large community forest covers a substantial portion of the remaining land.

Outside of a few small villages, most households lie scattered around the VDC, connected by dirt roads. Many families live in clusters of households, often adjoined by other households from the paternal extended family. As many young men (i.e. fathers) were working abroad for long periods of time, children often lived in households composed of their siblings, mother, paternal grandparents, and other paternal relatives (i.e. mother's in-laws). (The most recent census in Nepal (Central Bureau of Statistics (2011)), found a 1:2 male-to-female sex ratio in Meghauli for the 25-29 year-old age range.)

According to daily schedule reviews and community observations, children in Meghauli spent the majority of their time in or near their household or school each day. In their households on weekdays, after waking children washed, ate breakfast, went to school, and returned home in the afternoon. Most parents reported that their children spent the late afternoon and evening near their home, assisting the family with household chores, doing homework, eating, or playing with friends who live nearby. Reports were generally consistent with community observations, in which children were most often noted to be at or near a household doing cooking or cleaning chores, self-care routines, or playing with similar-aged children; in school (though several children were seen out of school during regular hours); playing sports in a community space (e.g., school yard or open field); working in fields; or accompanying an adult relative to town.

In discussions about girls, household work duties were especially prominent, as evident in this teacher's response about 14-15 year-old girls' daily routines:

The daughters (girls), now, after they wake up in the morning, now, let us say, they mostly focus in cleanliness. They wake up in the morning, go to toilet. Some of them also cook food and clean the house and yard, go to school and study. This is all. They go back home and clean the house. The daughters help the mother the most. They do their homework. This is all. And they sleep. Female teacher

Children in Meghauli typically began attending school at age 4 or 5, and school attendance rates were high in Meghauli, for both boys and girls. Most children attended public schools near their homes, though a large minority attended private schools in the area, occasionally at a greater distance from their home. Classes were divided into same-age, mixed gender classrooms with approximately 30-40 children per room. During community visits on school days, we occasionally observed school-aged children playing near their homes. (When asked, parents occasionally

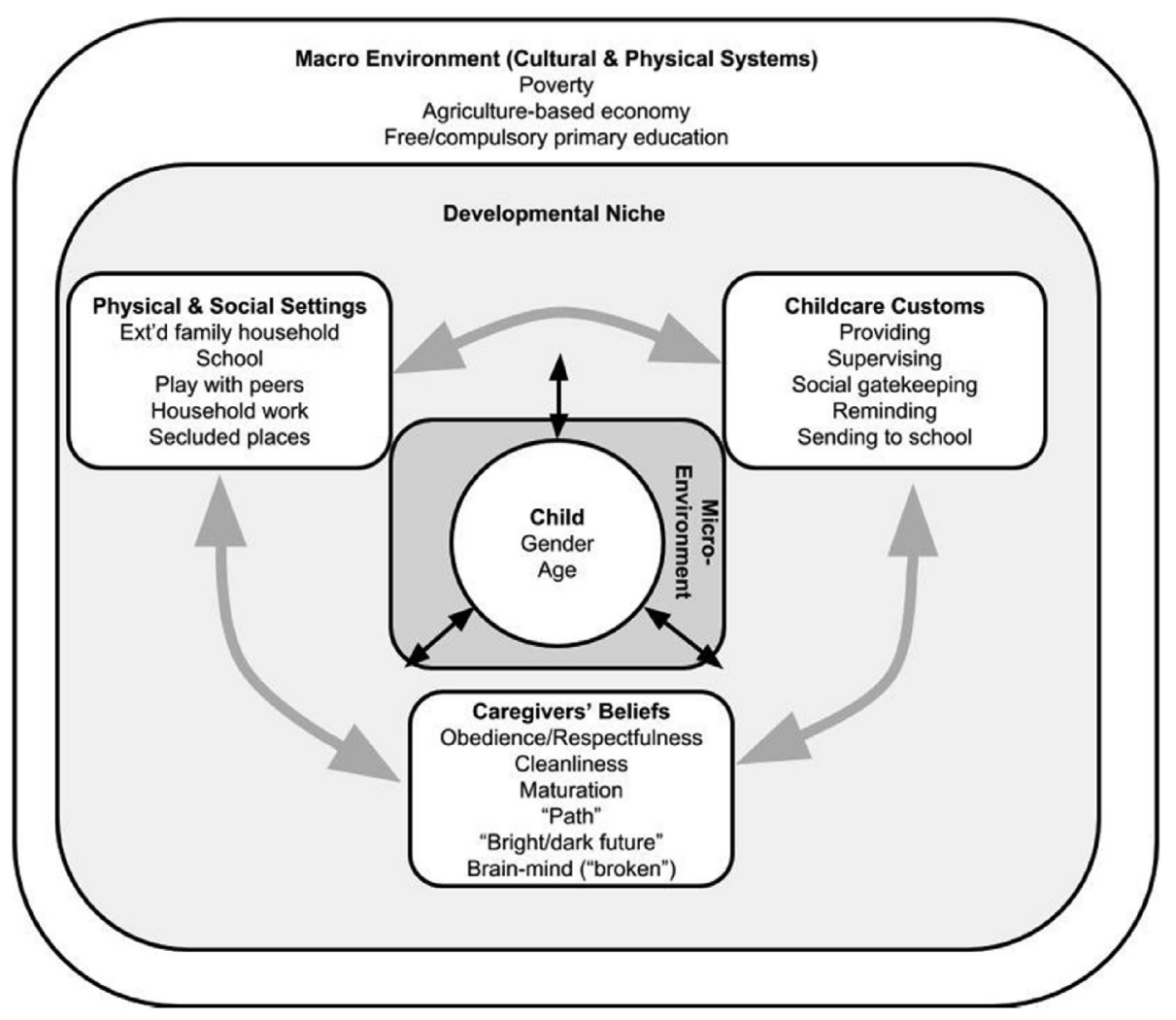

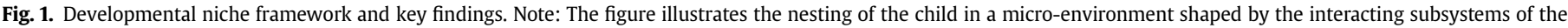

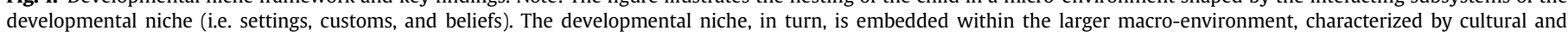

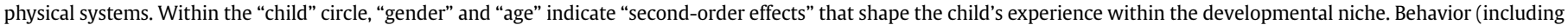
more and less problematic behavior) develops through the interactions of the child within the developmental niche over time.

Source: Based on concepts from Super and Harkness (1986) as illustrated in Worthman (2010b), adapted to include findings from the present study in Nepal. 
indicated that the child refused to attend.)

After school and on weekends, children were often observed playing together in public places. Younger children often played near their homes with siblings and neighbors, and appeared to favor groups of children of around the same age (e.g., within 2-4 years of their age). Older children, especially boys, were frequently seen playing sports-cricket, soccer, or volleyball-in open fields or on the school grounds after school. Younger teenagers were occasionally observed walking together or eating a snack at a neighborhood kiosk (usually in groups shortly after school let out), but were infrequently observed without adults in public spaces after dark.

\subsubsection{Childcare customs and practices}

Respondents highlighted several shared customs and practices that parents and other family caregivers in Meghauli routinely employed in rearing school-aged children. Three of the central practices that multiple respondents emphasized were: providing for basic needs, monitoring and social "gatekeeping", and supporting school success.

First, parents spent much of their time in efforts aimed at meeting their children's basic needs for nutrition, shelter, and other material needs (e.g., supplies for school.) Taking care of these needs often entailed working long hours away from their children, and "providing" was seen as parents' paramount responsibility, especially among poorer families for whom hunger was a constant threat.

Second, parents, teachers, and other elder family members and neighbors noted a shared responsibility to monitor their children's whereabouts and serve as "gatekeepers" for their children's social interactions. While parents often were the ones who monitored children, grandparents, aunts, uncles, and older siblings also participated in these efforts. In cases where fathers were living overseas, they often attempted to remain involved in supervision by talking frequently to their children by phone and by issuing directives through adults in the household.

Third, enrolling children in school and ensuring their regular attendance was a critical institutionalized practice in Meghauli, as it is in much of contemporary Nepal. Free and compulsory primary education was extended up to grade 8 in Nepal in 2009 (United Nations Educational, Scientific, and Cultural Organization [UNESCO], 2011). Net primary school enrollment in Nepal in 2009 was $96 \%$ for boys and $91 \%$ for girls, while secondary school enrollment was 74\% for boys and 66\% for girls (UNESCO, 2011).

Parents, teachers, and community leaders in Meghauli all emphasized the high priority they placed on school attendance and academic success. Parents and other caregivers were involved in schooling their children by sending them off to school daily, assisting them with homework in the evenings, and liaising with teachers when problems arose. On the other hand, parents also noted tensions between the ideal of promoting children's academic success and the practical daily demands of household work.

Most of all we want (children) to study well and be a good person. This is all. But, as we have needs, so they should (also) work ... They have to clean the house, sweep it, cook food and again after this. In some circumstances even my daughter has to bring the book and study and be in the kitchen. She has to study, do homework and also to cook the food. They also have to do this. - Father

\subsubsection{Parental ethnotheories}

Parents, teachers, and other caregivers described several concepts about the "nature" and "needs" of children, goals and expectations for their children's behavior, and beliefs about effective rearing techniques. Several participants described children as being on a "path" from a young age that was continuous into at least young adulthood and led to a "bright future" (Nepali: ujjwala bhavishya) or a "dark future" (amdhyaro bhavishya). The concept of a "bright future" encapsulated many of parents' long-term desires for their children and was most often described as a constellation of successes in academics, career, family relations, and social standing. In contrast, parents referred a "dark future" as one filled with failure, shame, poverty, and division from the family and community. Moreover, children's behavior and achievements also had repercussions for the family's social prestige or status (Nepali: ijjat) and even the community's (or caste's) reputation to outsiders.

In order to attain a "bright future" and avoid a "dark future", parents conveyed the concept of a "path" in which future success or failure began in early childhood. Parents related their socialization goals for children to indicators of which path a child was on and also to steps children should take to secure a bright future. Along these lines, parents and teachers noted that it was important for children to be respectful towards elders, obedient, and timely, and to complete their household chores and schoolwork. The majority of adults interviewed (17 out of 24) also noted that a key responsibility of children was to care for their personal hygiene and cleanliness.

In tension with the metaphor or a continuous path, children's nature and behavior were also described as changing and modifiable. Specifically, several respondents referred to maturation processes that predictably occurred over developmental time. Maturation was described as a process of growth toward greater awareness about appropriate behavior and greater capacity to reason. These changes were located by multiple participants in the dimaag (translation: brain-mind or social-mind), which has previously been described as the part of the self that is the seat of thoughts and social behavior (Kohrt and Harper, 2008), and in this study was described as "maturing" over time.

Parents, teachers, and other adult respondents in our study spoke at length about rearing strategies to promote change in children's behavior. In general, adults and children noted a preference for verbal reminders (Nepali: samjhaune) over disciplinary actions (especially physical punishment) in their approaches to shaping children's behavior. (See discussion below for additional details.)

\subsection{Defining behavior problems in the developmental niche}

Parents largely defined behavior problems in the context of daily role expectations, socialization goals, and long-term goals for their children. When children refused or failed to complete the tasks expected of them, or when their behavior seemed to threaten the attainment of parent's long-term goals for them, the behaviors were described as "problematic". For example, parents' most commonly expressed concerns about their children's behavior were related to not completing their expected household chores or self-care routines. Similar sets of behavioral concerns were also noted by several teachers.

The unnecessary things are not doing the work of the school, not studying. They should not do such work (i.e. behavior) and roaming round unnecessarily, not coming home in time, not eating in time and not studying in time is the mischievous act. -Male teacher

Likewise, failing to adequately maintain personal hygiene was commonly cited as an important problem. For example, one teacher commented: 
In home, we wish the children to go home after the school, wash their hands and feet, eat snacks and then start to study. But after they come back home they do not concentrate in hygiene and eat snacks without washing their hands and feet and they go to play running, I do not like this. - Female teacher

Parents also defined behaviors as problematic when they were perceived to carry imminent physical or social risks. Parents were concerned when what children did or where they went could lead to physical harm or ill health. While not always explicitly linked, some parents related their concerns about hygiene to risk for illness. Features of the physical environment, such as nearby rivers with strong currents, were forbidden places:

Last year a child died by drowning in the river as he went to catch the fish. The river is near here. Since then we yelled (at them) and now, they do not go to that river, if not then [long sigh]. - Mother/Female Community Health Volunteer

Parents were not only concerned about physical danger. Social danger loomed as at least as big a threat for children's bad behavior. For example, in pile-sort interviews when explaining why they placed "stealing" in the "worst" category, multiple respondents noted both the risk of physical injury from reprisal and also the damage to the family's social prestige (Nepali: ijjat). The family's prestige was not only at risk from gross misconduct but also from more basic adherence to socialization goals:

If the teachers tell us our child is (misbehaving) then we scold them telling them not to do so. 'Be good as the children of others are good by being educated. Do not ruin the prestige (ijjat) of the family. Study well-we are working hard for your education. Father

Children who consistently misbehaved were often labeled with the stigmatizing terms badmaash (translation: naughty) or bigrieko (literal translation: broken; refers to broken behavior, e.g., socially undesirable behavior) (see Supplemental Table 1 for additional Nepali terms for behavior problems.)

\subsection{Developing behavior problems in the developmental niche}

The developmental niche analysis highlighted salient risk processes for developing behavior problems, including poverty and predictable periods of unsupervised time. One of the prominent risk periods noted was the supervision "gap" that many families experienced during planting and harvest seasons. During planting and harvest, field laborers were often required to work from early in the morning (before school starts) until late in the evening. In many cases, all the adults in a household worked in the fields, leaving the children at home without adult supervision for several hours a day.

Parents expressed tension between the competing roles of providing for their family's financial needs and tending to their children's academic, emotional, and behavioral needs. However, they noted that they felt no choice but to prioritize the family's basic nutritional and material needs.

All the parents are involved in agriculture. They are farmers. They have to go to work from the morning-the parents go to work after cooking and eating. They have to work. We do not get to eat without working. This is the slum area. (Sigh.) If the children do not obey should we look after the children or go to work to fulfill the hand to mouth? This is also one of the problems here. - Mother

Despite parents' efforts to earn money and grow food for their families, poverty and food insecurity were reported as frequent experiences of children in Meghauli. When children were hungry or did not have the school supplies they needed, they were believed to be more vulnerable to misbehaving. Several respondents suggested that there was a direct connection between the type of need and behavior problems that resulted from it. For example, hungry children were described as being more likely to steal food; children who lacked basic school supplies were seen as likely to steal or skip school.

\subsection{Mitigating behavior problems in the developmental niche}

Parents attempted to mitigate behavior problems and the risks they carried by providing for children's physical needs; closely monitoring their children's location, activities, and social interactions; and utilizing a verbal "reminding" (samjhaune) strategy. These concepts were rooted in beliefs about the nature and needs of children and effective rearing strategies, and addressed the perceived risks from the physical and social surroundings in the developmental niche. Moreover, children were seen as being more or less responsive to change efforts depending on their developmental age and gender. That is, younger children and girls were typically seen as being more responsive to correction and guidance, whereas older boys were described as less sensitive to persuasion and requiring of more drastic measures (e.g., calling the police).

Providing food and physical needs (e.g., clothes, shelter, and school supplies) was seen not only as a general parental responsibility, but also as an important way parents could defend against problems such as stealing and skipping school. This reference to "providing" as a mitigation strategy appeared to be closely related to the perception that lack of food and basic materials predisposed children to misbehavior (see section above). Multiple community members described their efforts to remedy the behavior problems of children from other (poor) families by feeding them or providing basic school supplies.

After their basic needs were met, parents made efforts to protect their children from dangerous places and perceived negative social influences by monitoring their children and directing where and with whom children spent time. Caregivers monitored their children by being physically present in the household, keeping children (especially younger children and girls) close to the household during non-school hours, inquiring about their children's activities, and directing their children's activities (often toward household chores, especially in the case of older girls). Peers were seen as a potential negative influence, and parents attempted to prevent their children from affiliating with a "bad circle" of peers. Parents took a number of approaches to direct their children's interactions, including asking their children to account for their whereabouts, expecting them to come home immediately after school, and forbidding children perceived to be negative influences from entering their household.

The boys of my neighbor who were studying along with our children also use drugs. My son used to say that he does not like to be with his friend as his friend used to talk strangely when they were studying in class 10 . He could not say it to his friend. I used to tell (his friend) not to come into my home saying that he does not care about his studies. I used to tell him to go away. His parents also do not care about the child. The friends of my children say that I am harsh [alternate translation: stern]. 
Though they said it for sometime, but my children are good now. - Mother

When asked about appropriate and helpful responses to behavior problems, almost all interview participants (22 out of 24) and members of each FGD noted that parents should begin by "reminding" (Nepali: samjhaune) misbehaving children. The Nepali word "samjhaune" implies "reminding," "explaining," or "persuading" and was described as a way of addressing misbehavior by informing a child of his/her wrongdoing and reminding $\mathrm{him} /$ her of the future consequences of continuing the behavior. The examples respondents provided about samjhaune often demonstrated how elders reminded children of potential negative future outcomes of their behavior in order to persuade them to change their present behavior. The focus of discussions in samjhaune was usually future-oriented, and focused on negative consequences of behavior, as this teacher succinctly describes:

First, the school admonishes the children if they have done bad by saying not to do this and that. We should show the future perspective and inform them that their life will be ruined. Female teacher

For some, admonishing also included reminding a child of his/ her responsibility to his/her family to maintain their social standing (ijjat) and for the financial investment they have made in the child's education.

In addition to warnings about the future, samjhaune was also used to describe providing positive messages and setting positive expectations for a child. A teacher explained the importance of focusing on a child's good behavior when admonishing him:

We could admonish him saying that: "Little boy, you have done good work here so if you do this well then that's better." We should admonish him but not hate him or discard him. - Male teacher

In addition to concepts about how to address behavior problems, adult stakeholders also expressed shared ideas about when to best address them. Multiple informants noted that younger children's behavior could be changed more easily (compared with older children) through persuasion or punishment. Behavioral patterns were seen as increasingly resistant to caregivers' efforts to change them as they matured. Many parents reported less optimism when confronting behavior problems in older adolescents and used more severe interventions, including involving the police, or reported giving up efforts to change. Multiple informants reported that age 15 was as a critical threshold when the dimaag (brain-mind), and also bad behaviors, became more fixed and less amenable or likely to change.

Children up to 15 years, their brain-mind (dimaag) is not matured. So these children of 8-15 years, we can correct them on these things and-except these other bad things like alcohol and all-we cannot. - Mother

\subsection{Summary of findings}

Our findings demonstrate how child behavioral development is situated within context-specific physical and social settings, childcare customs, and caregiver's beliefs about the nature of children and effective child-rearing strategies (see Fig. 1). Each of these factors interacts with the others to form the "developmental niche", and child-specific factors (especially age and gender) help to determine which settings, customs, and beliefs the child is exposed to or influenced by. In turn, the developmental niche is influenced by larger macro-social level factors, such as larger patterns of poverty, national education policy, and regional geography and economic activities (e.g., agriculture in Meghauli).

\section{Discussion}

The findings from this study provide an initial step toward understanding the influences of the physical, social, and symbolic contexts of child development on locally meaningful definitions of behavior problems. Investigations of local settings, practices, and systems of meaning helped to make sense of the significance of behavior problems to caregivers, illuminated potential contextspecific risk and resilience processes, and identified local mitigation strategies that have potential for applications in interventions. The findings from our study illustrate some of the limitations of universal definitions and causal models that view behavior problems as the result of endogenous, biologically determined pathological processes that can be understood apart from the settings in which children develop and act (Panter-Brick, 1998; Singh, 2011).

In this study, parents', teachers' and peers' identified a consistent set of daily role expectations that varied somewhat according to the child's age, gender, and family's economic position. Respondents also identified patterns of child behavior that were concerning to them. Their concerns about child behavior problems were related to anticipated consequences to the child's immediate safety (e.g., fears of reprisal); the child's future prospects for academic and economic success, marriage, and personal reputation (characterized by participants as a "bright" vs. "dark" future); and the family's social prestige (ijjat) (Kohrt and Harper, 2008). Behavioral patterns that threatened to bring any of these consequences were considered to be problematic and requiring of intervention from parents or other older family members, teachers, or neighbors. The types of behaviors that were expected to bring consequences, the severity of those consequences, and fitting responses were substantially different for boys compared with girls. The findings from this study suggest that the developmental niche may be a useful framework for understanding how ecocultural contextual factors influence differential definitions, expressions of, and responses to child behavior problems across settings. Moreover, an ecocultural perspective is likely to enhance understanding of behavior problems in any setting.

Our findings suggest that school-age boys and girls in rural Nepal have different developmental experiences (e.g. role expectations, settings frequented, disciplinary experiences) that may affect their behavioral development. Our findings suggest potential mechanisms of differential socialization of child behavior by gender that could help to explain observed epidemiologic differences (Canino et al., 2010; Loeber et al., 2000). Gender-based differences in developmental experiences and their relationship to type and amount of behavior problems may be a fruitful target for future research in other settings.

Our qualitative study has also suggested potential risk and protective processes relevant to low- and middle-income country (LMIC) settings that could be evaluated in future research. For example, there has been little research about the relationship between poverty and child behavior problems outside of highincome, Western settings (Loeber et al., 2000; Zilanawala et al., 2015). Our findings suggest that poverty may be a risk factor in the rural Nepali setting, and also suggests potential pathways for this effect (e.g. decreased parental availability for supervision.) Similarly, unsupervised time after school during planting and harvest seasons emerged as a potential risk period. Interventions could 
target this risk period through season-specific afterschool tutoring or activities or by identifying community resources for childcare and supervision.

Our study also suggests that already existing local childcare customs and practices, and concepts such as samjhaune in rural Nepal, might be a useful source for readily acceptable and potentially effective interventions. Interventions drawing on local concepts of causation and effective behavioral change may be more culturally compelling than strategies imported from high-income countries (Panter-Brick et al., 2006). Previous studies in LMIC have demonstrated the feasibility of using systematic approaches to integrate local qualitative findings with existing evidence-based practice elements to develop effective interventions for behavior problems (Jordans et al., 2011). In Nepal, interventions building on existing concepts of verbal reminders and guidance contained within the samjhaune concept have the potential to be more culturally compelling and could utilize existing local knowledge and expertise. For example, local samjhaune "experts" could help coach teachers and parents in applying this concept in the classroom or at home.

Our study was limited by heavy reliance on self-report data. We also included only limited data from children, who may have been able to provide additional information about influences of peer interactions and an experiential perspective on other ecocultural factors. The age range examined may have also presented too much developmental variability to make firm conclusions about narrower age ranges. Future studies may beneficially examine the concepts about behavior problems presented in our study in the context of lived experience of children and families affected by behavior problems.

Finally, it is worth pointing out that our study's focus on shared settings, customs, and beliefs resulted in a de-emphasis on individual child characteristics. While our approach intentionally departed from endogenous child factors in an attempt to draw attention to the role of ecocultural factors, ultimately a synthesis of individual and contextual influences will provide a more complete, informative, and useful model for understanding and addressing problems in child behavioral development. Worthman's (2010b) bioecocultural model is an example of a recent synthetic model that could be applied in future studies to elucidate how individual children's "needs, capacities, and sensitivity to environmental input" (p. 555) are differentially shaped by ecocultural contextual factors.

\section{Conclusions}

The findings of our study illustrate the transactional nature of behavior problem development that involves context-specific goals, roles, and concerns that are likely to affect adults' interpretations and responses to children's behavior. Our findings also demonstrate how physical and social settings can create opportunities for or barriers to developing behavior problems. The developmental niche offers an analytic framework that is useful for understanding cross-cultural variability in the definitions of, distributions of, and responses to child behavior problems. Greater attention to the ecocultural context of development in studies of child psychopathology may help guide the development of more coherent definitions and more acceptable and effective intervention strategies.

\section{Acknowledgements}

The authors thank all the individuals who participated in this study for their time and their willingness to invite us into their homes, schools, and communities. We thank Transcultural
Psychosocial Organization (TPO) Nepal for their administrative support of this project. We appreciate Sarah McIvor-Murray's critical feedback on the manuscript.

\section{Appendix A. Supplementary data}

Supplementary data related to this article can be found at http:// dx.doi.org/10.1016/j.socscimed.2016.04.020.

\section{References}

Achenbach, T.M., Edelbrock, C.S., 1983. Manual for the Child Behavior Checklist and Revised Child Behavior Profile. Department of Psychiatry of the University of Vermont.

Adhikari, R.P., Upadhaya, N., Gurung, D., Luitel, N.P., Burkey, M.D., Kohrt, B.A., Jordans, M.J., 2015. Perceived behavioral problems of school aged children in rural Nepal: a qualitative study. Child Adolesc. Psychiatry Ment. Health 9 (25), 1-9. http://doi.org/10.1186/s13034-015-0061-8.

American Psychiatric Association, 2013. Diagnostic and Statistical Manual of Mental Disorders, DSM-5 (Arlington, VA).

Arnett, J.J., 2008. The neglected 95\%: why American psychology needs to become less American. Am. Psychol. 63 (7), 602.

Bronfenbrenner, U., 1979. The Ecology of Human Development: Experiments by Nature and Design. Harvard University Press, Cambridge, MA.

Bronfenbrenner, U., 2005. Making Human Beings Human: Bioecological Perspectives on Human Development. Sage.

Canino, G., Polanczyk, G., Bauermeister, J.J., Rohde, L.A., Frick, P.J., 2010. Does the prevalence of $\mathrm{CD}$ and $\mathrm{ODD}$ vary across cultures? Soc. Psychiatry Psychiatr. Epidemiol. 45 (7), 695-704.

Central Bureau of Statistics, 2011. National Population and Housing Census 2011. Government of Nepal, Kathmandu, Nepal.

Cole, P.M., Tamang, B.L., 1998. Nepali children's ideas about emotional displays in hypothetical challenges. Dev. Psychol. 34 (4), 640-646.

Cole, P.M., Tamang, B.L., Shrestha, S., 2006. Cultural variations in the socialization of young children's anger and shame. Child. Dev. 77 (5), 1237-1251.

Crijnen, A.A., Achenbach, T.M., Verhulst, F.C., 1997. Comparisons of problems reported by parents of children in 12 cultures: total problems, externalizing, and internalizing. J. Am. Acad. Child. Adolesc. Psychiatry 36 (9), 1269-1277. http:// doi.org/10.1097/00004583-199709000-00020.

Furlong, M., McGilloway, S., Bywater, T., Hutchings, J., Smith, S.M., Donnelly, M. 2012. Behavioural and Cognitive-behavioural Group-based Parenting Programmes for Early-onset Conduct Problems in Children Aged 3 to 12 Years (Wiley Online Library).

Goodman, R., 1997. The strengths and difficulties questionnaire: a research note. J. Child Psychol. Psychiatry 38 (5), 581-586.

Harkness, S., Super, C.M., 1996. Parents Cultural Belief Systems: Their Origins, Expressions, and Consequences. Guilford Press, New York.

Jordans, M.J., Tol, W.A., Komproe, I.H., 2011. Mental health interventions for children in adversity: pilot-testing a research strategy for treatment selection in lowincome settings. Soc. Sci. Med. 73 (3), 456-466. http://doi.org/10.1016/j. socscimed.2011.06.004.

Kessler, R.C., Angermeyer, M., Anthony, J.C., de Graaf, R., Demyttenaere, K., Gasquet, I., Haro, J.M., 2007. Lifetime prevalence and age-of-onset distributions of mental disorders in the World health Organization's World mental health survey initiative. World Psychiatry 6 (3), 168.

Kirmayer, L.J., Swartz, L., 2013. Culture and global mental health. In: Patel, V., Prince, M., Cohen, A., Minas, H. (Eds.), Global Mental Health: Principles and Practice. Oxford University Press.

Kleinman, A.M., 1977. Depression, somatization and the "new cross-cultural psychiatry". Soc. Sci. Med. 11 (1), 3-9.

Kohrt, B.A., Harper, I., 2008. Navigating diagnoses: understanding mind-body relations, mental health, and stigma in Nepal. Cult. Med. Psychiatry 32 (4), 462-491. http://doi.org/10.1007/s11013-008-9110-6.

Kohrt, B.A., Maharjan, S.M., 2009. When a child is no longer a child: Nepali ethnopsychology of child development and violence. Stud. Nepali Hist. Soc. 14 (1), $107-142$.

Lambert, M.C., Weisz, J.R., Knight, F., Desrosiers, M.F., Overly, K., Thesiger, C., 1992. Jamaican and American adult perspectives on child psychopathology: further exploration of the threshold model. J. Consult. Clin. Psychol. 60 (1), 146.

Lau, A.S., 2006. Making the case for selective and directed cultural adaptations of evidence-based treatments: examples from parent training. Clin. Psychol. Sci. Pract. 13 (4), 295-310.

LeVine, R.A., New, R.S., 2008. Anthropology and Child Development: A Crosscultural Reader. Blackwell Pub.

Loeber, R., Burke, J.D., Lahey, B.B., Winters, A., Zera, M., 2000. Oppositional defiant and conduct disorder: a review of the past 10 years, part I. J. Am. Acad. Child Adolesc. Psychiatry 39 (12), 1468-1484.

Lund, C., Tomlinson, M., De Silva, M., Fekadu, A., Shidhaye, R., Jordans, M., Prince, M. 2012. PRIME: a programme to reduce the treatment gap for mental disorders in five low-and middle-income countries. PLoS Med. 9 (12), e1001359.

Panter-Brick, C., 1998. Biosocial Perspectives on Children, vol. 10. Cambridge 
University Press.

Panter-Brick, C., Clarke, S.E., Lomas, H., Pinder, M., Lindsay, S.W., 2006. Culturally compelling strategies for behaviour change: a social ecology model and case study in malaria prevention. Soc. Sci. Med. 62 (11), 2810-2825.

Patel, S.A., Murray-Kolb, L.E., LeClerq, S.C., Khatry, S.K., Tielsch, J.M., Katz, J., Christian, P., 2013. Household wealth and neurocognitive development disparities among school-aged children in Nepal. Paediatr. Perinat. Epidemiol. 27 (6), 575-586.

Patel, V., Sumathipala, A., 2001. International representation in psychiatric literature Survey of six leading journals. Br. J. Psychiatry 178 (5), 406-409.

Patterson, G.R., 2002. The Early Development of Coercive Family Process. American Psychological Association, Washington, D. C.

OSR International, 2012. NVivo (Version 10). QSR International Pty Ltd.

Sameroff, A., 2009. The Transactional Model. American Psychological Association, Washington, D.C.

Singh, I., 2011. A disorder of anger and aggression: children's perspectives on attention deficit/hyperactivity disorder in the UK. Soc. Sci. Med. 73 (6), 889-896.

Super, C.M., Harkness, S., 1986. The developmental niche: a conceptualization at the interface of child and culture. Int. J. Behav. Dev. 9 (4), 545-569.

Super, C.M., Harkness, S., 2002. Culture structures the environment for development. Hum. Dev. 45 (4), 270-274.

Tobin, G.A., Begley, C.M., 2004. Methodological rigour within a qualitative framework. Methodol. Issues Nurs. Res. 48 (4), 388-396.
United Nations Development Programme, 2009. Nepal Human Development Report 2009.

United Nations Educational, Scientific, and Cultural Organization, 2011. World Data on Education. Retrieved from. http://unesdoc.unesco.org/images/0021/002113/ 211309e.pdf.

Weisz, J.R., McCarty, C.A., Eastman, K.L., Chaiyasit, W., Suwanlert, S., 1997. Developmental psychopathology and culture: ten lessons from Thailand. In: Luthar, S.S. (Ed.), Developmental Psychopathology: Perspectives on Adjustment Risk, and Disorder: Cambridge University Press, Cambridge, UK, pp. 568-592.

Whiting, J.W., 1977. A model for psychocultural research. Cult. Infancy Var. Hum. Exp. 29-48.

Woolfenden, S., Williams, K., Peat, J., 2001. Family and parenting interventions in children and adolescents with conduct disorder and delinquency aged 10-17. Cochrane Database Syst. Rev. 2.

World Health Organization, 2010. International Classification of Disease (ICD-10 (Geneva).

Worthman, C.M., 2010a. Formative Experiences: The Interaction of Caregiving Culture, and Developmental Psychobiology. Cambridge University Press.

Worthman, C.M., 2010b. The ecology of human development: evolving models for cultural psychology. J. Cross Cult. Psychol. 41 (4), 546-562.

Zilanawala, A., Sacker, A., Nazroo, J., Kelly, Y., 2015. Ethnic differences in children's socioemotional difficulties: findings from the Millennium Cohort Study. Soc. Sci. Med. 134, 95-106. 